\title{
Accelerated extracellular matrix turnover during exacerbations of COPD
}

Jannie M. B. Sand ${ }^{1,2^{*}}$, Alan J. Knox³, Peter Lange ${ }^{2,4}$, Shu Sun ${ }^{1}$, Jacob H. Kristensen ${ }^{1}$, Diana J. Leeming ${ }^{1}$, Morten A. Karsdal' ${ }^{1}$, Charlotte E. Bolton ${ }^{3+}$ and Simon R. Johnson ${ }^{3+}$

\begin{abstract}
Background: Exacerbations of chronic obstructive pulmonary disease (COPD) contribute significantly to disease progression. However, the effect on tissue structure and turnover is not well described. There is an urgent clinical need for biomarkers of disease activity associated with disease progression. Extracellular matrix (ECM) turnover reflects activity in tissues and consequently assessment of ECM turnover may serve as biomarkers of disease activity. We hypothesized that the turnover of lung ECM proteins were altered during exacerbations of COPD.

Methods: 69 patients with COPD hospitalised for an exacerbation were recruited at admission and returned for a 4 weeks follow-up. Competitive ELISAs measuring circulating protein fragments in serum or plasma assessed the formation and degradation of collagen types III (Pro-C3 and C3M, respectively), IV (P4NP 7S and C4M, respectively), and VI (Pro-C6 and C6M, respectively), and degradation of elastin (ELM7 and EL-NE) and versican (VCANM).

Results: Circulating levels of C3M, C4M, C6M, ELM7, and EL-NE were elevated during an exacerbation of COPD as compared to follow-up (all $P<0.0001$ ), while VCANM levels were decreased $(P<0.0001$ ). Pro-C6 levels were decreased and P4NP 7S levels were elevated during exacerbation $(P<0.0001)$. Pro- $C 3$ levels were unchanged. At time of exacerbation, degradation/formation ratios were increased for collagen types $\mathrm{II}$ and $\mathrm{VI}$ and decreased for collagen type IV.

Conclusions: Exacerbations of COPD resulted in elevated levels of circulating fragments of structural proteins, which may serve as markers of disease activity. This suggests that patients with COPD have accelerated ECM turnover during exacerbations which may be related to disease progression.
\end{abstract}

Keywords: COPD, Exacerbation, Extracellular matrix, Tissue turnover, Collagens, Elastin, Versican, Disease activity, Biomarkers

\section{Background}

Chronic obstructive pulmonary disease (COPD) is a heterogeneous, slow progressing disease characterized by persistent airflow limitation resulting from chronic inflammation, structural changes, and small airway narrowing [1]. The main structural proteins of the extracellular matrix (ECM) of the lung are collagens, elastin, and proteoglycans. ECM turnover is part of healthy tissue maintenance, where old proteins are

\footnotetext{
* Correspondence: jsa@nordicbioscience.com

${ }^{\dagger}$ Equal contributors

${ }^{1}$ Nordic Bioscience, Biomarkers and Research, Herlev Hovedgade 207, 2730

Herlev, Denmark

${ }^{2}$ Section of Social Medicine, Institute of Public Health, University of

Copenhagen, Oester Farimagsgade 5, 1014 Copenhagen K, Denmark

Full list of author information is available at the end of the article
}

degraded and new proteins formed [2]. However, excessive ECM turnover may drive the structural changes in COPD and promote loss of lung function. A key challenge in COPD is the identification of biomarkers of disease progression [3]. ECM investigation by assessment of lung structural proteins may provide biomarkers of disease activity and prognosis. Novel technologies assessing ECM protein turnover are promising candidate tools $[4,5]$.

Exacerbations are periods of increased disease activity that drive COPD progression by accelerating loss of lung function [6], reducing quality of life [7], and causing mortality [8]. Patients in all COPD stages may experience exacerbations, although they become more frequent with increasing disease severity [9]. It

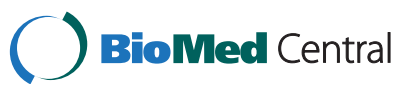


is difficult to predict their occurrence, and the best predictor of future exacerbations is an exacerbation history $[9,10]$. Although exacerbations are key events in COPD pathogenesis, little is known regarding structural changes in lung tissue during these events. Matrix metalloproteinase 9 (MMP-9) levels are known to be elevated while tissue inhibitor of metalloproteinase 1 (TIMP-1) levels are decreased in sputum of COPD patients at time of exacerbation compared to stable COPD [11], suggesting a destructive environment. In the present work we tested the hypothesis that ECM turnover, assessed systemically by biomarkers of protein degradation and formation, would be accelerated during an exacerbation of COPD where disease activity is high.

\section{Methods}

Study design

Patients presenting with a hospital admission deemed by a medical consultant to be a COPD exacerbation during 2011 and 2012 were recruited within $24 \mathrm{~h}$ of admission. All patients provided written informed consent, and the study was approved by the National Research Ethics Service (10/H0403/85). Blood samples were collected at time of exacerbation and at recovery: a 4 week follow-up visit performed a median of 30 (IQR 28-34) days after admission. At follow-up visit, the patients underwent standard post-bronchodilator spirometry, and performed a six minute walking distance (6MWD). Patient-reported measures included assessments of dyspnoea, using the Medical Research Council (MRC) dyspnoea scale, at follow-up visit, and smoking history. The inclusion criterion was a clinical diagnosis of acute COPD exacerbation at hospital admission made by a consultant physician. A physician diagnosis or radiological evidence of pneumonia was an exclusion criterion. The study comprised 69 patients with paired samples and with airflow obstruction (ratio of forced expiratory volume in one second $\left(\mathrm{FEV}_{1}\right)$ to forced vital capacity $(\mathrm{FVC})$ of $<0.7)$ confirmed at follow-up visits.

\section{Biomarkers}

Serum and plasma samples were stored at $-80{ }^{\circ} \mathrm{C}$ until analysed. C3M, C4M, Pro-C3, P4NP 7S, ELM7, and EL-NE, were measured in serum, while C6M, Pro-C6, and VCANM were measured in plasma. All assays employed a monoclonal antibody directed against either a protein fragment produced by protease cleavage during degradation or formation, or an internal protein sequence. An overview of the assays used in this study and their technical specifications is given in Table 1. All samples were measured within the detection range of each assay and any sample with values below the lower limit of detection (LLOD) was assigned the value of LLOD.

\section{Statistical analysis}

The main outcome predictor was change in circulating levels of C3M, C4M, C6M, Pro-C3, P4NP 7S, Pro-C6, ELM7, EL-NE, and VCANM. Based on previous data for these assays, for $90 \%$ power to detect a $20 \%$ difference in biomarker levels between exacerbation and follow-up with a two-sided $\alpha$ of 0.05 , a minimum of 27 to 55 patients were required. Biomarker levels were normalised by log-transformation. Exacerbation and follow-up values were compared by paired $t$-test. Univariate and multivariate linear regressions were performed using the log-transformed biomarker data to identify associations with clinical parameters. The multivariate analysis included age, gender, body mass index (BMI), smoking pack years, and smoking status as additional explanatory variables. $P<0.05$ was considered significant. Analyses were performed using MedCalc Statistical Software v.12 (MedCalc Software, Ostend, Belgium).

Table 1 Overview of the assays used in this study to assess extracellular matrix turnover

\begin{tabular}{|c|c|c|c|c|c|}
\hline Assay & Target & $\begin{array}{l}\text { Detection range } \\
(\mathrm{ng} / \mathrm{ml})\end{array}$ & $\begin{array}{l}\text { Intra-and inter-assay } \\
\text { variation (\%) }\end{array}$ & Reference level (ng/ml), mean (SD) & References \\
\hline $\mathrm{C} 3 \mathrm{M}$ & Collagen type III degraded by MMPs & $5.52-177$ & 3.4 and 9.8 & $15.3(3.8)$ & [28] \\
\hline $\mathrm{C} 4 \mathrm{M}$ & Collagen type IV degraded by MMPs & $22.8-748$ & 4.2 and 18.5 & $55.4(17.8)$ & [29] \\
\hline C6M & Collagen type VI degraded by MMPs & $4.88-420$ & 8.0 and 11.0 & $8.85(5.1)$ & {$[30]$} \\
\hline ELM7 & Elastin degraded by MMP-7 & $1.16-36.6$ & 8.1 and 9.1 & $2.23(0.74)$ & Preliminary data \\
\hline EL-NE & Elastin degraded by neutrophil elastase & $1.76-167$ & 8.6 and 12.9 & $4.09(2.24)$ & {$[31]$} \\
\hline VCANM & Versican degraded by MMPs & $0.78-7.13$ & 3.0 and 7.6 & $1.20(0.23)$ & [32] \\
\hline Pro-C3 & Collagen type III propeptide (N-terminal) & $5.32-96.4$ & 6.5 and 12.4 & $12.3(4.4)$ & [33] \\
\hline P4NP 7S & Collagen type IV 75 domain & $32.9-3460$ & 9.4 and 14.2 & $263(91.3)$ & [34] \\
\hline Pro-C6 & Collagen type VI C5 domain & $0.30-117$ & 4.8 and 15.2 & $4.37(0.69)$ & Preliminary data \\
\hline
\end{tabular}

The reference level was provided by the manufacturer (Nordic Bioscience) and refers to the biomarker level of a healthy population in the relevant matrix, i.e. serum (C3M, C4M, Pro-C3, P4NP 7S, ELM7, EL-NE) or plasma (C6M, Pro-C6, VCANM). SD, standard deviation; MMP, matrix metalloproteinase 


\section{Results}

\section{Basic demographics}

Patient demographics and clinical characteristics are summarised in Table 2. Patients were mostly men (71 \%) and ex-smokers (55\%). They were hospitalised for a median [IQR] of 3 [2-6] days, and follow-up visit was performed at 30 [28-34] days after admission.

\section{Protein turnover during COPD exacerbation}

Circulating levels of protein fragments released at time of exacerbation and at a clinically stable disease period at 4 weeks follow-up are presented in Table 3. Degradation fragments of collagen type III (C3M), collagen type IV $(\mathrm{C} 4 \mathrm{M})$, collagen type VI $(\mathrm{C} 6 \mathrm{M})$, and elastin (ELM7 and EL-NE) were significantly elevated at exacerbation compared to follow-up (all $P<0.0001$; Fig. 1a-e). In contrast, a fragment of versican degradation (VCANM) showed a significantly decreased mean level at time of exacerbation $(P<0.0001$; Fig. $1 f)$. Levels of fragments related to protein formation were not significantly changed for collagen type III (Pro-C3), but were increased for collagen type IV (P4NP 7S; $P<0.0001$ ) and decreased for collagen type VI (Pro-C6; $P<0.0001$ ) at exacerbation compared to follow-up (Fig. 1g-i). To investigate the effect of smoking on circulating levels of protein fragments, analysis was performed on current and ex smokers, individually, with similar results (data not shown).

\section{Collagen degradation/formation balance}

The balance between degradation and formation of collagens was investigated by calculating the ratio between

Table 2 Basic characteristics of the COPD population at follow-up visit 4 weeks after exacerbation onset

\begin{tabular}{ll}
\hline Variable & Patients $(n=69)$ \\
\hline Age (years), median (IQR) & $67(61-75)$ \\
Female sex, n (\%) & $20(29)$ \\
BMI (kg/m²) & $25.7(6.3)$ \\
Current smokers, n (\%) & $31(45)$ \\
Smoking pack years (years) & $52(26)$ \\
Length of hospitalisation (days), median (IQR) & $3(2-6)$ \\
FEV 1 (liters) & $1.19(0.50)$ \\
FEV 1 (\% of predicted) & $45.8(16.1)$ \\
FVC (liters) & $2.55(0.81)$ \\
FVC (\% of predicted) & $77.5(19.0)$ \\
FEV 1 /FVC ratio & $0.46(0.11)$ \\
$6 M W D$ (meters) & $166(119)$ \\
MRC dyspnoea score, median (IQR) & $4(3-4)$
\end{tabular}

Variables are listed as mean (standard deviation) unless otherwise stated. IQR, interquartile range; $\mathrm{BMI}$, body mass index; $\mathrm{FEV}_{1}$, forced expiratory volume in one second; FVC, forced vital capacity; 6MWD, 6 min walking distance; MRC, Medical Research Council
Table 3 Biomarker levels at exacerbation and 4 weeks follow-up

\begin{tabular}{llll}
\hline & Exacerbation $(\mathrm{ng} / \mathrm{ml})$ & Follow-up $(\mathrm{ng} / \mathrm{ml})$ & $P$ value \\
\hline C3M & $29.24[26.32-32.49]$ & $22.64[20.78-24.67]$ & $<0.0001$ \\
C4M & $95.96[85.83-107.28]$ & $73.30[66.59-80.69]$ & $<0.0001$ \\
C6M & $19.78[16.82-23.27]$ & $13.27[11.56-15.23]$ & $<0.0001$ \\
ELM7 & $4.26[3.90-4.64]$ & $3.61[3.37-3.87]$ & $<0.0001$ \\
EL-NE & $7.79[6.30-9.63]$ & $5.23[4.41-6.21]$ & $<0.0001$ \\
VCANM & $1.69[1.58-1.80]$ & $1.87[1.78-1.97]$ & 0.0001 \\
Pro-C3 & $12.10[10.60-13.81]$ & $12.79[11.35-14.42]$ & 0.2549 \\
P4NP 7S & $510.99[440.91-592.21]$ & $359.20[312.28-413.17]$ & $<0.0001$ \\
Pro-C6 & $5.36[4.81-5.99]$ & $6.38[5.71-7.14]$ & $<0.0001$ \\
\hline
\end{tabular}

Results are presented as geometric mean [95 \% confidence interval] and corresponding $\mathrm{P}$ values comparing circulating biomarker levels at time of exacerbation and follow-up

fragments of degradation and formation for collagen types III, IV, and VI (Fig. 2). The mean degradation/formation ratio $[95 \% \mathrm{CI}]$ was significantly elevated at time of exacerbation for collagen type III (2.33 [2.03-2.66] vs. 1.72 [1.51-1.96], $P<0.0001)$ and collagen type VI $(3.61$ [2.86-4.56] vs. 2.00 [1.64-2.44], $P<0.0001)$. In contrast, the collagen type IV degradation/formation ratio was $0.18[0.17-0.20]$ at exacerbation and increased to 0.20 [0.19-0.22] at follow-up $(P=0.0008)$.

\section{Associations with clinical parameters}

At follow-up, C3M was positively associated with BMI $(r=0.245, P=0.049)$, Pro-C6 was positively associated with age $(r=0.317, P=0.022)$, and EL-NE was positively associated with white blood cell count $(r=0.279, P=0.022)$. No associations were seen with smoking pack years, MRC score, length of hospitalisation, or sputum production. Pro-C3 levels were positively associated with $\mathrm{FEV}_{1} \%$ of predicted value (\%pred) and FVC \%pred, and these remained significant after correcting for age, gender, BMI, smoking pack years, and smoking status (Table 4). 6MWD was negatively associated with $\mathrm{C} 3 \mathrm{M}, \mathrm{C} 4 \mathrm{M}, \mathrm{C} 6 \mathrm{M}$, and P4NP 7S (Table 4). Following correction for age, gender, BMI, smoking pack years, and smoking status, associations with $\mathrm{C} 3 \mathrm{M}$ and $\mathrm{C} 6 \mathrm{M}$ remained significant (Table 4).

\section{Discussion}

To our knowledge, this is the first study describing that COPD exacerbations are associated with accelerated tissue turnover by measuring circulating levels of ECM protein fragments during and after an exacerbation. These results indicate that the processing of structural proteins in the lungs is altered during severe exacerbations as compared to a clinically stable disease period at 4 weeks follow-up. The main findings were; 1) significantly elevated levels of circulating 


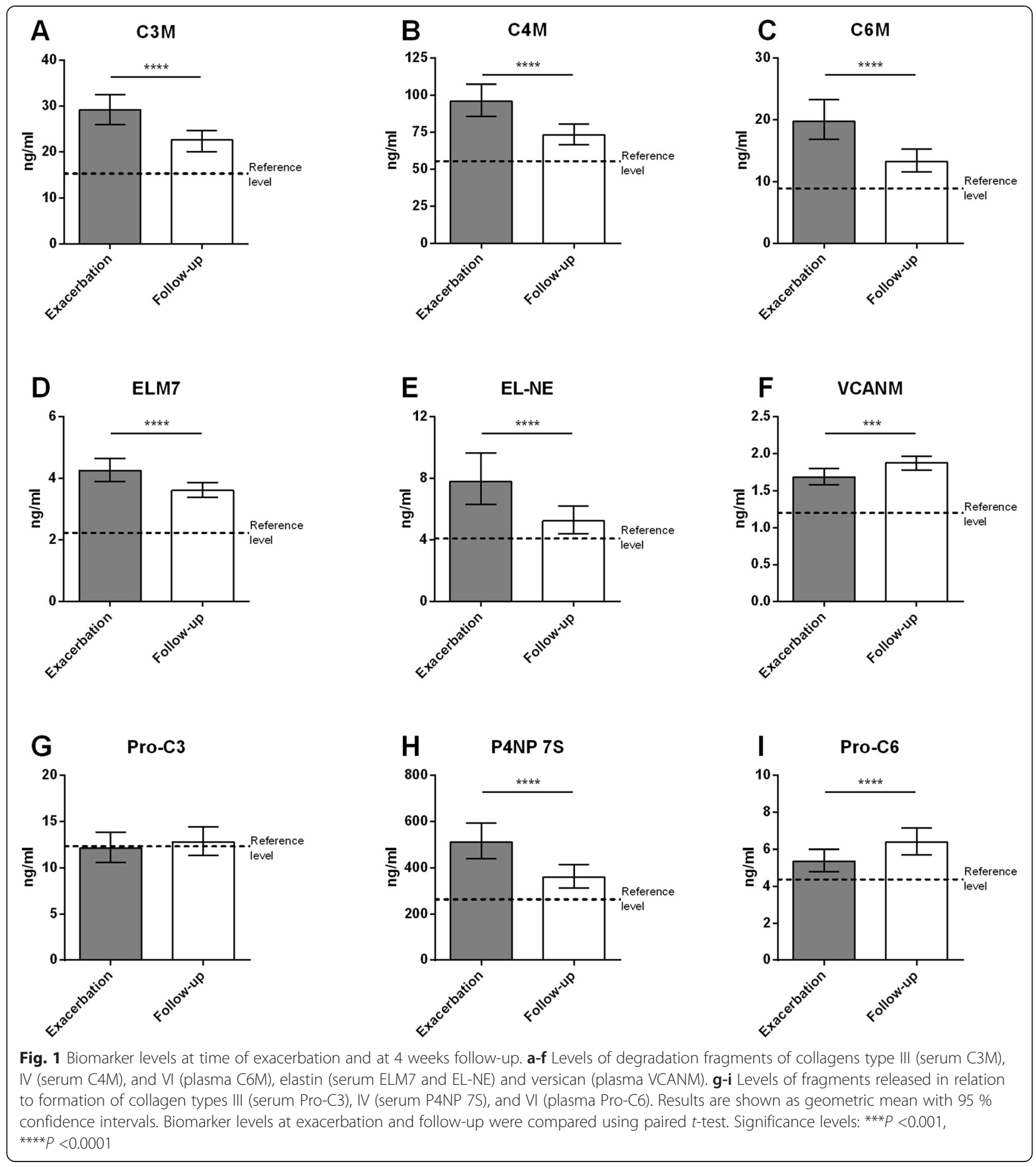

biomarkers of collagen types III (C3M), IV (C4M), and VI (C6M), and elastin (ELM7 and EL-NE) degradation were found at time of exacerbation as compared to follow-up; 2) at exacerbation, levels of circulating biomarkers released in relation to protein formation was significantly elevated for type IV collagen, whereas it was significantly decreased for type VI collagen, and unchanged for type III collagen; 3) the tissue turnover balance, assessed systemically by the collagen degradation/formation ratio, was increased for type III and VI collagen and decreased for type IV collagen; 4) circulating levels of Pro-C3 were associated with disease severity, assessed by $\mathrm{FEV}_{1}$ \%pred and FVC \%pred, while C3M, C4M, C6M, and P4NP 


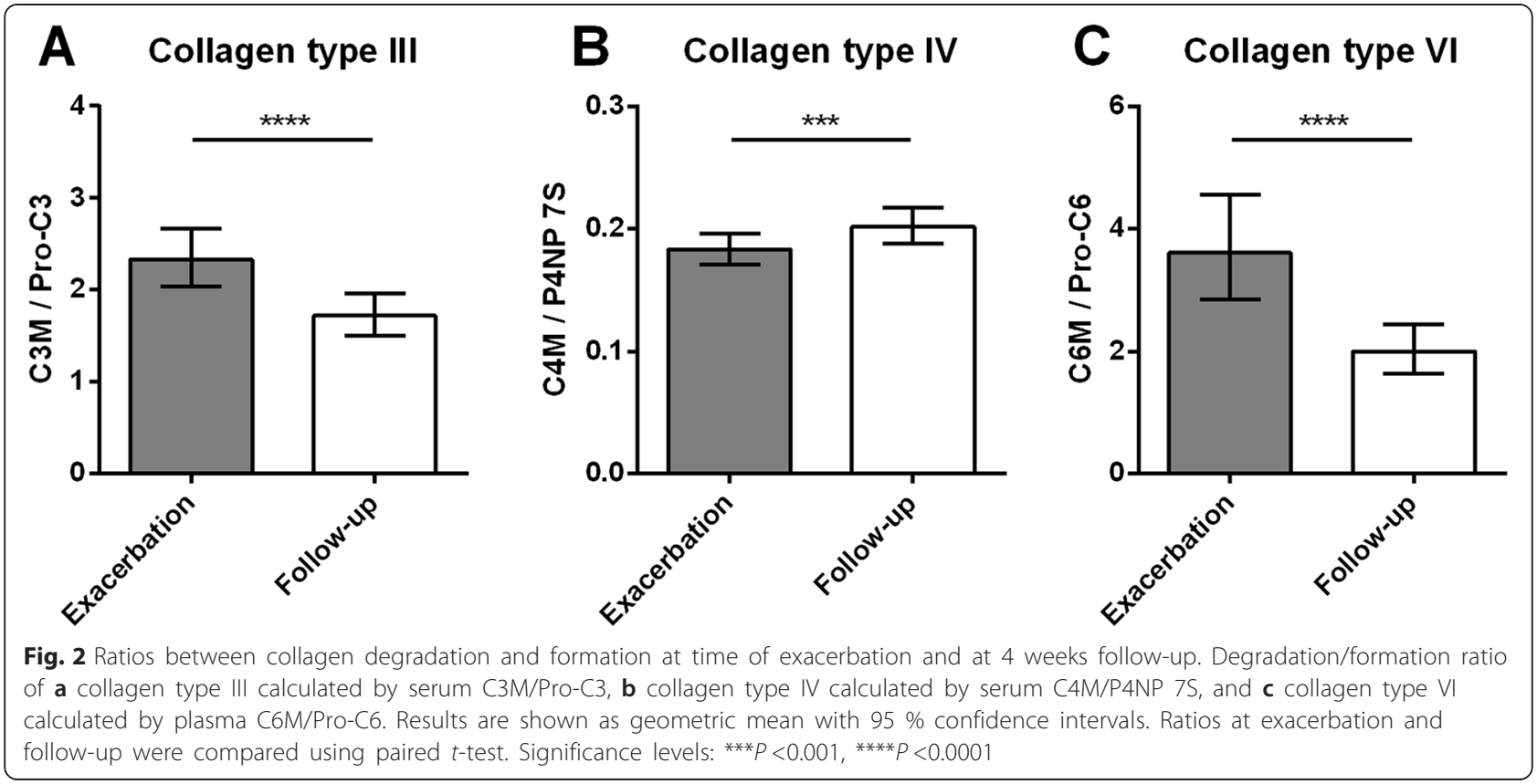

$7 \mathrm{~S}$ were associated with exercise capacity, assessed by 6MWD.

Structural ECM proteins are crucial for maintaining tissue function. Collagens provide flexibility and form a scaffold for cells, elastin provides tissue elasticity, and proteoglycans such as versican maintain ECM integrity by interacting with hyaluronan. Others have investigated the collagen turnover by different technologies. O'Reilly et al. reported elevated sputum proline-glycine-proline (PGP), a neutrophil chemoattractant derived from collagen degradation, around the time of exacerbation in a small cohort of COPD patients [12]. Further, azithromycin treatment

Table 4 Associations between biomarker levels and clinical parameters

\begin{tabular}{llll}
\hline & FEV $_{1} \%$ pred & FVC \%pred & $6 \mathrm{MWD}$ \\
\hline C3M & -0.118 & -0.201 & $-0.350^{* *}\left(-0.311^{*}\right)$ \\
C4M & -0.149 & -0.161 & $-0.289^{*}(-0.252)$ \\
C6M & -0.096 & -0.226 & $-0.403^{* *}\left(-0.354^{* *}\right)$ \\
ELM7 & -0.090 & -0.149 & -0.178 \\
EL-NE & -0.039 & -0.106 & -0.186 \\
VCANM & -0.0004 & -0.080 & -0.067 \\
Pro-C3 & $0.345^{*}\left(0.320^{*}\right)$ & $0.313^{*}\left(0.305^{*}\right)$ & 0.055 \\
P4NP 7S & -0.086 & -0.193 & $-0.269^{*}(-0.230)$ \\
Pro-C6 & 0.023 & -0.046 & -0.153 \\
\hline
\end{tabular}

Results are presented as univariate correlation coefficients ( $r$ ) for each marker. In brackets are given multivariate correlation coefficients for markers showing significant $r$. The multivariate linear regression analysis included age, gender, $\mathrm{BMI}$, smoking pack years, and smoking status as additional explanatory variables. Significance levels: ${ }^{*} P<0.05,{ }^{*} P<0.01$. FEV 1 , forced expiratory volume in one second; \%pred, percentage of predicted value; FVC, forced vital capacity; 6MWD, 6 min walking distance targeting exacerbations reduced sputum PGP. Their results indicate that COPD exacerbation causes collagen destruction, resulting in PGP release. As the PGP motif exists in several ECM proteins, especially collagens [13], and its sputum levels reflect overall lung ECM degradation; we investigated ECM turnover further by analysing different proteins. Further, our study utilised samples from the same patients 4 weeks apart, providing insight into short-term changes in protein turnover. We previously reported elevated biomarker levels, including serum C3M and C6M, in a small cohort of mild COPD patients compared to healthy controls [5]. Our previous study utilised early research and development versions of some of the assays presented here, resulting in somewhat different levels, but nevertheless indicating that measuring protein fragments in the circulation of patients with COPD may be of interest.

Collagens are the most abundant components of the lung interstitium, and fibrillar collagens such as types I and III maintain the lung architecture. Collagen type IV is the main constituent of the basement membrane, a sheet-like ECM structure underlying epithelial and endothelial cells. Collagen type VI molecules form anchoring fibrils that can interact with collagen types I and IV and fibronectin thereby adhering to the interstitial matrix and basement membrane, upholding tissue structure [14]. Our results indicate that both the interstitial matrix and the basement membrane were turned over faster during an exacerbation and point to a destructive environment affecting several structural proteins. Both formation and degradation of collagen types III, IV, and VI were changed. Significantly decreased plasma Pro-C6 levels during a COPD exacerbation suggest that collagen type VI formation was lower than 
during stable disease. Consistent with this, the collagen type VI degradation/formation ratio was significantly elevated during exacerbation when compared to follow-up, indicating a balance shift towards collagen type VI degradation during exacerbation. As collagen type VI is involved in reconstructing damaged tissue by stimulating fibroblast proliferation and preventing apoptosis [15-17], its enhanced degradation during exacerbation may contribute to COPD progression. It should be noted that the process of proteins and peptides entering the circulation is not fully understood. Different peptides may diffuse from the lung tissue to the circulation in different manners. Thus, the degradation/formation ratios cannot be interpreted directly but may serve as an indication of the shift in the balance between degradation and formation at time of exacerbation and follow-up. The ratio as such does not provide any information on the degree of protein formation and degradation. Collagen type III formation was not affected by exacerbation as shown by unaltered levels of serum Pro-C3. However, degradation (serum C3M) and degradation/formation ratio were significantly elevated during exacerbation, suggesting an overall collagen type III destruction during these episodes. As collagen type III is an abundant lung ECM protein, its destruction may greatly alter tissue function. Interestingly, serum Pro-C3 was the only biomarker directly associated with $\mathrm{FEV}_{1}$ \%pred, indicating a relation to disease severity rather than activity. Levels of serum C4M and P4NP 7S were significantly increased during exacerbation compared to followup. This indicated that both formation and degradation of collagen type IV were elevated during exacerbation, resulting in an accelerated turnover rate. The balanced outcome of this turnover showed a decreased degradation during exacerbation compared to follow-up, as shown by the C4M/P4NP 7S ratio. C3M, C4M, C6M, and P4NP 7S were negatively associated with $6 \mathrm{MWD}$, indicating an interesting relationship between exercise capacity and collagen turnover that should be investigated further.

Elastin degradation by MMP-7 and neutrophil elastase was increased during exacerbation, as shown by elevated levels of serum ELM7 and EL-NE, respectively. Elastin provides lungs with elasticity, allowing movement during breathing and preservation of the lung structure and function. Neutrophil elastase is released by neutrophils which are abundant during tissue inflammation [18], and degradation of elastin by neutrophil elastase (EL-NE) may reflect the local inflammatory response. Neutrophil elastase activity towards fibrinogen and the proportion of blood neutrophils are elevated during a COPD exacerbation compared to stable disease $[19,20]$, demonstrating a role of neutrophil elastase in exacerbations. Our data support this finding by showing increased activity of neutrophil elastase towards elastin at time of exacerbation. Although our results for ELM7 and EL-NE were similar, it is important to recognize the difference in the assays and the value of knowing exactly what epitope the antibody targets [21].

Interestingly, plasma VCANM was decreased during exacerbation, implying that versican processing is different to other ECM proteins. Versican degradation is linked to vascular diseases, suggesting that proteolytic modifications have pathophysiological implications for the vascular system [22]. However, versican also seems to play an important role in the lungs and versican content in the lungs is increased in COPD patients compared to controls [23, 24]. The increased versican content is believed to inhibit the function of elastin binding protein (EBP), thus impairing formation of elastic fibers [25]. An increased versican content in the lungs was associated with decreased elastin and EBP content and a decrease in $\mathrm{FEV}_{1}$ [23] supporting a different role for versican compared with elastin and collagens in COPD.

Exacerbations of COPD are periods of increased disease activity, when the biological processes that drive disease progression may be accelerated [26]. While $\mathrm{FEV}_{1}$ is a measure of disease severity it is not a good measure of disease activity, and a low $\mathrm{FEV}_{1}$ does not necessarily reflect a fast disease progression [3]. The rate of decline in $\mathrm{FEV}_{1}$ represents a better measure of disease activity or disease progression $[3,27]$. However, measuring the rate of decline in $\mathrm{FEV}_{1}$ requires very large and long-term studies, making it impractical for assessment of new disease-modifying treatments [3]. The recent ATS/ERS statement on research questions in COPD proposes measures of lung tissue destruction as potential biomarkers of disease activity and recommends further studies on disease activity biomarkers [26]. The results presented here indicate that biomarkers of lung ECM turnover may be markers of disease activity as circulating levels are elevated during exacerbation. These markers assess protease-generated protein fragments, also known as protein fingerprints [21], derived from tissue. The assays used are very sensitive, allowing measurements of low abundance protein fragments in circulation. This provides an indirect measure of tissue turnover in the lung, which may reflect disease activity. This assessment may better reflect dynamic remodelling of lung tissue compared to spirometry measures, which in the stable state reflect the cumulative disease process and reflect both the baseline level and the additional bronchoconstriction during the acute exacerbation. Future studies on the ECM turnover in patients classified as slow or fast progressors may reveal if patients with higher disease activity have elevated biomarker levels also in the stable state. Reliable markers of disease activity are an urgent need in the field of COPD as they have the potential to improve and shorten the assessment of new treatments [3]. 
A limitation to this study was that biomarkers assessed systemically may originate from multiple organs, thus introducing a background level from other tissues. However, the turnover in healthy tissue is balanced and occurs at a slow pace with release of low levels of protein fragments. In this study we compared circulating levels in a patient group at two time points, minimising the risk that observed differences are not the cause of the exacerbation. Future studies should assess the ECM turnover in sputum for comparison, eliminating the extra-pulmonary contribution. Another limitation was that treatment for exacerbation may influence protein turnover. However, irrespective of treatment this study showed accelerated ECM turnover during exacerbation. This study represents daily clinical practice for COPD patients admitted to hospital for an exacerbation, thus it includes patients on different treatment regimens and with exacerbation from different causes. Further, comorbidities may influence biomarker levels and the lack of information on these is a limitation, however the paired design of this study may eliminate some of the effect. Although patients did not have evidence of myocardial infarction or other significant events during hospitalisation, we cannot exclude that subclinical events may have occurred affecting the levels of the measured biomarkers. Future studies should explore the effect of treatment and co-morbidities on ECM turnover as well as exacerbation aetiology.

\section{Conclusions}

We have shown that during an acute exacerbation of COPD there is an accelerated turnover of ECM proteins relevant to lung. The enhanced protein turnover is likely to be important in COPD progression. Assessment of these biomarkers may provide useful endpoints in proofof-concept studies aiming at reducing lung tissue turnover during exacerbations.

\footnotetext{
Abbreviations

\%pred: Percentage of predicted value; 6MWD: Six minutes walking distance; BMl: Body mass index; Cl: Confidence interval; COPD: Chronic obstructive pulmonary disease; EBP: Elastin binding protein; ECM: Extracellular matrix; $\mathrm{FEV}_{1}$ : Forced expiratory volume in one second; FVC: Forced vital capacity; IQR: Interquartile range; LLOD: Lower limit of detection; MMP: Matrix metalloproteinase; MRC: Medical research council; PGP: Proline-glycine-proline; SD: Standard deviation; TIMP: Tissue inhibitor of metalloproteinase.
}

\section{Competing interests}

JMBS, SS, JHK, DJL, and MAK are full-time employees at Nordic Bioscience. MAK is a stockholder of Nordic Bioscience. CEB and SRJ report grants from the Medical Research Council, UK in relation to this work. SRJ reports personal fees from Novartis, not related to the current manuscript. AJK and $\mathrm{PL}$ declare no competing interests in relation to the current manuscript. $\mathrm{PL}$ reports grants and personal fees from GlaxoSmithKline, Novartis, Boehringer Ingelheim, and Almirall, and personal fees from Sandoz, Mundipharma, Takeda, AstraZeneca, and Pfizer, not related to the current manuscript.

\section{Authors' contributions}

JMBS had primary responsibility for the statistical analyses, data interpretation, and manuscript preparation. DJL, MAK, CEB, and SRJ contributed to data interpretation. AJK, CEB, and SRJ designed the study, recruited the samples needed for measurements as well as the clinical data. JMBS, AJK, PL, SS, JHK, DJL, MAK, CEB, and SRJ all contributed with important intellectual content and writing the manuscript. All authors read and approved the final manuscript.

\section{Acknowledgements}

In addition to Nottingham University Hospitals Trust, we would like to thank Derby Hospital Trust (Dr G Lowrey) and Sherwood Forest Hospitals Trust (Dr A Molyneux) for recruiting samples from patients with COPD. Further, we acknowledge the funding from the Medical Research Council, UK; the Danish Agency for Science, Technology and Innovation; and the Danish Research Foundation supporting this work. The funders of the study had no role in the study design, data collection, data analysis, data interpretation, writing of the manuscript, or the decision to publish.

\section{Author details}

${ }^{1}$ Nordic Bioscience, Biomarkers and Research, Herlev Hovedgade 207, 2730 Herlev, Denmark. ${ }^{2}$ Section of Social Medicine, Institute of Public Health, University of Copenhagen, Oester Farimagsgade 5, 1014 Copenhagen K, Denmark. ${ }^{3}$ Division of Respiratory Medicine and Nottingham Respiratory Research Unit, University of Nottingham, Hucknall Road, Nottingham NG5 1 PB, UK. ${ }^{4}$ Section of Respiratory Medicine, Hvidovre Hospital, Kettegaards Alle 30, 2650 Hvidovre, Denmark.

Received: 20 January 2015 Accepted: 26 May 2015

Published online: 11 June 2015

\section{References}

1. Global strategy for the diagnosis, management, and prevention of COPD. Global initiative for chronic obstructive lung disease (GOLD) 2015. http://www.goldcopd.org/. Accessed 20 Apr 2015.

2. Cox TR, Erler JT. Remodeling and homeostasis of the extracellular matrix: implications for fibrotic diseases and cancer. Dis Model Mech. 2011;4:165-78

3. Vestbo J, Rennard S. Chronic obstructive pulmonary disease biomarker(s) for disease activity needed-urgently. Am J Respir Crit Care Med. 2010;182:863-4.

4. Karsdal MA, Nielsen MJ, Sand JM, Henriksen K, Genovese F, Bay-Jensen AC, et al. Extracellular matrix remodeling: the common denominator in connective tissue diseases. Possibilities for evaluation and current understanding of the matrix as more than a passive architecture, but a key player in tissue failure. Assay Drug Dev Technol. 2013;11:70-92.

5. Leeming DJ, Sand JM, Nielsen MJ, Genovese F, Martinez FJ, Hogaboam CM, et al. Serological investigation of the collagen degradation profile of patients with chronic obstructive pulmonary disease or idiopathic pulmonary fibrosis. Biomark Insights. 2012;7:119-26.

6. Donaldson GC, Seemungal TA, Bhowmik A, Wedzicha JA. Relationship between exacerbation frequency and lung function decline in chronic obstructive pulmonary disease. Thorax. 2002;57:847-52.

7. Seemungal TA, Donaldson GC, Paul EA, Bestall JC, Jeffries DJ, Wedzicha JA. Effect of exacerbation on quality of life in patients with chronic obstructive pulmonary disease. Am J Respir Crit Care Med. 1998;157:1418-22.

8. Soler-Cataluna JJ, Martinez-Garcia MA, Roman SP, Salcedo E, Navarro M, Ochando R. Severe acute exacerbations and mortality in patients with chronic obstructive pulmonary disease. Thorax. 2005;60:925-31.

9. Hurst JR, Vestbo J, Anzueto A, Locantore N, Mullerova H, Tal-Singer R, et al. Susceptibility to exacerbation in chronic obstructive pulmonary disease. N Engl J Med. 2010;363:1128-38.

10. Donaldson GC, Wedzicha JA. COPD exacerbations.1: epidemiology. Thorax. 2006;61:164-8

11. Mercer PF, Shute JK, Bhowmik A, Donaldson GC, Wedzicha JA, Warner JA. MMP-9, TIMP-1 and inflammatory cells in sputum from COPD patients during exacerbation. Respir Res. 2005;6:151.

12. O'Reilly PJ, Jackson PL, Wells JM, Dransfield MT, Scanlon PD, Blalock JE. Sputum PGP is reduced by azithromycin treatment in patients with COPD and correlates with exacerbations. BMJ Open. 2013;3, e004140.

13. Pfister RR, Haddox JL, Sommers Cl, Lam KW. Identification and synthesis of chemotactic tripeptides from alkali-degraded whole cornea. A study 
of $\mathrm{N}$-acetyl-proline-glycine-proline and N-methyl-proline-glycine-proline. Invest Ophthalmol Vis Sci. 1995;36:1306-16.

14. Engvall $\mathrm{E}$, Hessle $H$, Klier G. Molecular assembly, secretion, and matrix deposition of type VI collagen. J Cell Biol. 1986;102:703-10.

15. Ruhl M, Sahin E, Johannsen M, Somasundaram R, Manski D, Riecken EO, et al. Soluble collagen VI drives serum-starved fibroblasts through S phase and prevents apoptosis via down-regulation of Bax. J Biol Chem. 1999;274:34361-8.

16. Ruhl M, Johannsen M, Atkinson J, Manski D, Sahin E, Somasundaram R, et al. Soluble collagen VI induces tyrosine phosphorylation of paxillin and focal adhesion kinase and activates the MAP kinase erk2 in fibroblasts. Exp Cell Res. 1999;250:548-57.

17. Atkinson JC, Ruhl M, Becker J, Ackermann R, Schuppan D. Collagen VI regulates normal and transformed mesenchymal cell proliferation in vitro. Exp Cell Res. 1996;228:283-91.

18. Tetley TD. Inflammatory cells and chronic obstructive pulmonary disease. Curr Drug Targets Inflamm Allergy. 2005;4:607-18.

19. Corhay JL, Moermans C, Henket M, Nguyen DD, Duysinx B, Louis R. Increased of exhaled breath condensate neutrophil chemotaxis in acute exacerbation of COPD. Respir Res. 2014;15:115.

20. Carter RI, Ungurs MJ, Mumford RA, Stockley RA. Aalpha-Val360: a marker of neutrophil elastase and COPD disease activity. Eur Respir J. 2013:41:31-8.

21. Karsdal MA, Delvin E, Christiansen C. Protein fingerprints - relying on and understanding the information of serological protein measurements. Clin Biochem. 2011;44:1278-9.

22. Kenagy $\mathrm{RD}$, Plaas $\mathrm{AH}$, Wight TN. Versican degradation and vascular disease. Trends Cardiovasc Med. 2006;16:209-15.

23. Merrilees MJ, Ching PS, Beaumont B, Hinek A, Wight TN, Black PN. Changes in elastin, elastin binding protein and versican in alveoli in chronic obstructive pulmonary disease. Respir Res. 2008;9:41.

24. Hallgren O, Nihlberg K, Dahlback M, Bjermer L, Eriksson LT, Erjefalt JS, et al. Altered fibroblast proteoglycan production in COPD. Respir Res. 2010;1 1:55.

25. Huang R, Merrilees MJ, Braun K, Beaumont B, Lemire J, Clowes AW, et al. Inhibition of versican synthesis by antisense alters smooth muscle cell phenotype and induces elastic fiber formation in vitro and in neointima after vessel injury. Circ Res. 2006;98:370-7.

26. Celli BR, Decramer M, Wedzicha JA, Wilson KC, Agusti A, Criner GJ, et al. An official American thoracic society/European respiratory society statement: research questions in COPD. Eur Respir J. 2015:45:879-905.

27. Bihlet AR, Karsdal MA, Bay-Jensen AC, Read S, Kristensen JH, Sand JM, et al. Clinical drug development using dynamic biomarkers to enable personalized health care in Chronic Obstructive Pulmonary Disease. Chest. 2015 (in press).

28. Barascuk N, Veidal SS, Larsen L, Larsen DV, Larsen MR, Wang J, et al. A novel assay for extracellular matrix remodeling associated with liver fibrosis: an enzyme-linked immunosorbent assay (ELISA) for a MMP-9 proteolytically revealed neo-epitope of type III collagen. Clin Biochem. 2010;43:899-904.

29. Sand JM, Larsen L, Hogaboam C, Martinez F, Han M, Rossel LM, et al. MMP mediated degradation of type IV collagen alpha 1 and alpha 3 chains reflects basement membrane remodeling in experimental and clinical fibrosis-validation of two novel biomarker assays. PLoS One. 2013;8, e84934.

30. Veidal SS, Karsdal MA, Vassiliadis E, Nawrocki A, Larsen MR, Nguyen QH, et al. MMP mediated degradation of type VI collagen is highly associated with liver fibrosis-identification and validation of a novel biochemical marker assay. PLoS One. 2011;6, e24753.

31. Kristensen JH, Karsdal MA, Sand JM, Willumsen N, Diefenbach C, Svensson B, et al. Serological assessment of neutrophil elastase activity on elastin during lung ECM remodeling. BMC Pulm Med. 2015;15:53.

32. Barascuk N, Genovese F, Larsen L, Byrjalsen I, Zheng Q, Sun S, et al. A MMP derived versican neo-epitope is elevated in plasma from patients with atherosclerotic heart disease. Int J Clin Exp Med. 2013;6:174-84.

33. Nielsen MJ, Nedergaard AF, Sun S, Veidal SS, Larsen L, Zheng Q, et al. The neo-epitope specific PRO-C3 ELISA measures true formation of type III collagen associated with liver and muscle parameters. Am J Transl Res. 2013;5:303-15

34. Leeming DJ, Nielsen MJ, Dai Y, Veidal SS, Vassiliadis E, Zhang C, et al. Enzyme-linked immunosorbent serum assay specific for the $7 S$ domain of Collagen Type IV (P4NP 7S): A marker related to the extracellular matrix remodeling during liver fibrogenesis. Hepatol Res. 2012;42:482-93.

\section{Submit your next manuscript to BioMed Central and take full advantage of:}

- Convenient online submission

- Thorough peer review

- No space constraints or color figure charges

- Immediate publication on acceptance

- Inclusion in PubMed, CAS, Scopus and Google Scholar

- Research which is freely available for redistribution

Submit your manuscript at www.biomedcentral.com/submit 